\title{
Effect of quasi-periodic gravitational modulation on the convective instability in Hele-Shaw cell
}

\author{
T. Boulal $^{a *}$, S. Aniss $^{a}$, M. Belhaq $^{a}$, A. Azouani $^{b}$
}

April 26, 2008

${ }^{a}$ University Hassan II Ain-Chock, Faculty of Sciences, Laboratory of Mechanics, Casablanca, Morocco

${ }^{b}$ Institute of Mathematics I, Free University Berlin, Arnimallee 2-6, D-14195 Berlin, Germany

\begin{abstract}
The aim of the present paper is to examine the effect of a quasi-periodic gravitational modulation on the onset of convective instability in Hele-Shaw cell. The quasi-periodic modulation considered here consists in a modulation having two incommensurate frequencies. This study is an extension of a previous work by Aniss et al. [10] in which only a periodic gravitational modulation was considered. We have shown that for $\operatorname{Pr}=O(1)$ or $\operatorname{Pr}>>1$, the gravitational modulation has no effect on the convective threshold as expected. However, for $\operatorname{Pr}=O\left(\epsilon^{2}\right)$, it turns out that a modulation with two incommensurate frequencies has a stabilizing or a destabilizing effect strongly depending on the frequencies ratio.
\end{abstract}

\section{Introduction}

Several works have been devoted to analyzing the effect of periodic modulation on the stability of the motionless state of a heated liquid layer. This periodic modulation concerns either gravity or temperatures imposed on the horizontal planes of a liquid layer. The gravitational modulation, which can be realized by vertically oscillating horizontal liquid layer and its frontiers, acts on the entire volume of the liquid and has stabilizing or destabilizing effect depending on the amplitude and frequency of vibrations [1-10,13]. This effect was analyzed for a liquid layer heated from below or from above (stable or unstable equilibrium configurations). Here the onset of convection presents a competition between harmonic and subharmonic modes; see for instance $[3,8,9,12-16]$. A similar modulation to the gravitational one can be realized in a horizontal ferromagnetic liquid layer heated from above and submitted to a time-periodic external magnetic field instead of vertical vibrations $[11,12]$. The other type of modulation [14-25], in which the temperatures is oscillating at the boundaries, is mainly concentrated in a boundary layer whose thickness decreases with increasing frequency of modulation. Note that this modulation is similar to the gravitational one for low modulation frequencies. In the out of-phase oscillations case, the 
modulation presents a stabilizing effect. Nevertheless, the case corresponding to the in-phase oscillations shows a stabilizing effect for low frequencies and a destabilizing effect at high ones [4]. In contrast to the standard periodic modulation, the effect of a quasi-periodic gravitational modulation on the stability of a heated liquid layer with infinite extension in the horizontal directions was studied recently [26]. The present work focuses attention on the influence of a quasi-periodic gravitational modulation on the convective instability threshold in the case of a Hele-Shaw geometrical configuration. Here, we consider a Newtonian fluid layer confined in a horizontal Hele-Shaw cell and submitted to vertical quasi-periodic oscillations having two incommensurate frequencies. This motion can be realized, for instance, by using a coupled system of two blocks and two springs attached to a horizontal wall and oscillating vertically. One of the two blocks corresponds to the physical system under consideration. Neglecting frictions, the solutions of the linear system involve a quasi-periodic motion of two incommensurate frequencies. Following [11], a dimensional and an asymptotic analysis allow us to distinguish between two linear formulations. Each one of these formulations depends on the order of magnitude of the Prandtl number. The first formulation corresponds to $\operatorname{Pr}=O(1)$ or $\operatorname{Pr} \gg 1$ while the second one corresponds to $\operatorname{Pr}=O\left(\epsilon^{2}\right)$, where $\epsilon$ designates the aspect ratio of the cell. Therefore, we determine the stability criterion for these two asymptotic cases. In the first formulation, we have obtained, as expected, the same criterion of stability that the one of the unmodulated case. In contrast to the first asymptotic formulation, the governing system of equations corresponding to the second formulation is reduced to a damped quasi-periodic Mathieu equation. In this case, the Floquet theory cannot be applied to determine a stability criterion. However, following Rand et al. [27,28], we investigate the convective quasi-periodic parametric instability occurring in the second formulation and we determine the instability criterion in function of the problem parameters.

\section{Formulation}

Consider a Newtonian fluid confined in a horizontal Hele-Shaw cell of infinite extent in the $x$ direction; see Figure 1. Denote by $d$ the height of the cell, $e$ the distance between the vertical planes and $\epsilon=e / d$ the aspect ratio of the cell; the values $y= \pm e / 2$ and $z=0, d$, correspond to the boundaries of the cell. We assume that the fluid confined in the cell is bounded vertically by two thermally insulating walls and horizontally by two perfect heat conducting plates having respectively constant temperatures $T_{1}$ and $T_{2}\left(T_{1}>T_{2}\right.$ or $\left.T_{1}<T_{2}\right)$. Suppose that the HeleShaw cell is submitted to an oscillatory motion according to the law of displacement, $z=$ $\left[b_{1} \cos \left(\omega_{1} t\right)+b_{2} \cos \left(\omega_{2} t\right)\right] \mathbf{k}$, where $\omega_{1}$ and $\omega_{2}$ are two dimensional incommensurate frequencies. The parameters $b_{1}$ and $b_{2}$ are the amplitudes of motion and $\mathbf{k}$ is the unit vector upward. Therefore, the fluid layer is submitted to two volumic forces: the gravitational force field $\rho \mathbf{g}$ and the quasiperiodic one $-\rho\left[b_{1} \omega_{1}^{2} \cos \left(\omega_{1} t\right)+b_{2} \omega_{2}^{2} \cos \left(\omega_{2} t\right)\right] \mathbf{k}$. The equilibrium of the fluid layer corresponds to a rest state with a conductive regime. Under these assumptions, the linear system of the governing equations, corresponding to the perturbation of the equilibrium state, is given by the following Navier-Stokes equations in the Boussinesq approximation

$$
\nabla \cdot \mathbf{V}=0
$$




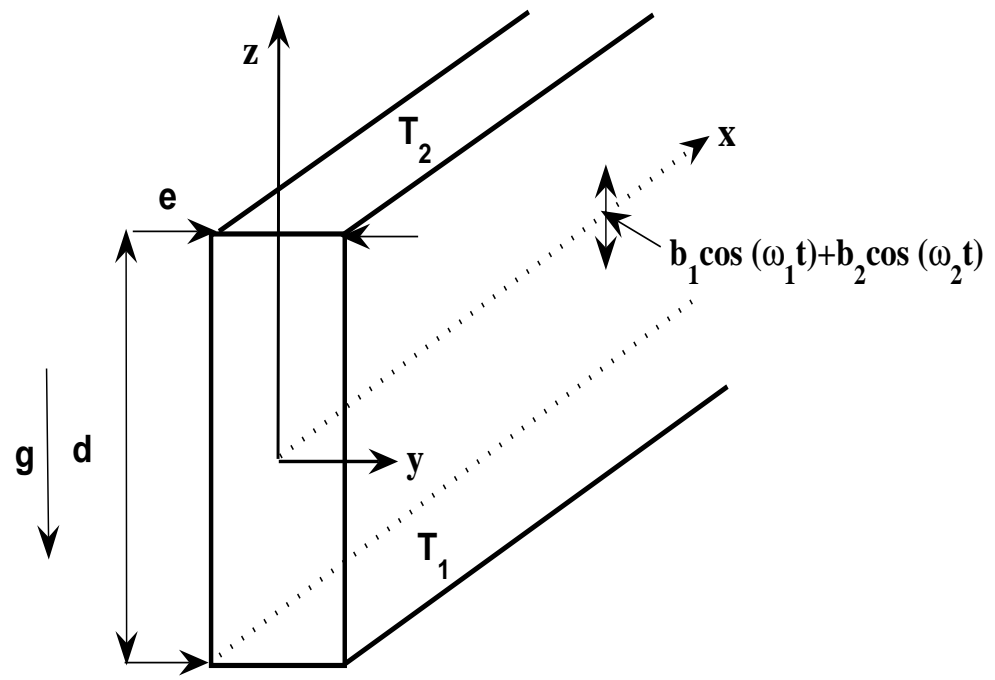

Figure 1: Hele-Shaw geometry 


$$
\begin{gathered}
\rho \frac{\partial \mathbf{V}}{\partial t}=-\nabla P+\mu \Delta \mathbf{V}+\rho \beta\left[g+b_{1} \omega_{1}^{2} \cos \left(\omega_{1} t\right)+b_{2} \omega_{2}^{2} \cos \left(\omega_{2} t\right)\right] T \mathbf{k} \\
\frac{\partial T}{\partial t}-\frac{T_{1}-T_{2}}{d} w=\kappa \Delta T
\end{gathered}
$$

where $\rho, \beta, \nu$ and $\kappa$ designate respectively, the density, the coefficient of thermal dilatation, the kinematic viscosity and the thermal diffusivity of the fluid. Here, we assume that

$$
b_{1} \omega_{1}^{2}=b_{2} \omega_{2}^{2}
$$

To introduce a perturbation parameter involving only the aspect ratio of the cell, $\epsilon$, we perform a dimensional analysis by means of an appropriate choice of scales used in convection problems in Hele-Shaw cell [29]. Thus, the time is scaled by $d^{2} / \kappa$, the coordinates $(x, y, z)$ are scaled by $(d, e, d)$, the velocity field $\mathbf{V}(u, v, w)$ is scaled by $\left(\kappa / d, e \kappa / d^{2}, \kappa / d\right)$, and the pressure and temperature are scaled by $\rho \nu \kappa / e^{2}$ and $\left(T_{1}-T_{2}\right)$, respectively. Hence the linear system of equations (1)-(3) is written as

$$
\begin{aligned}
& \frac{\partial u}{\partial x}+\frac{\partial v}{\partial y}+\frac{\partial w}{\partial z}=0 \\
& \epsilon^{2} \operatorname{Pr}^{-1} \frac{\partial u}{\partial t}=-\frac{\partial p}{\partial x}+\epsilon^{2} \triangle_{2} u+\frac{\partial^{2} u}{\partial y^{2}} \\
& \epsilon^{4} \operatorname{Pr}^{-1} \frac{\partial v}{\partial t}=-\frac{\partial p}{\partial y}+\epsilon^{4} \triangle_{2} v+\epsilon^{2} \frac{\partial^{2} v}{\partial y^{2}} \\
& \epsilon^{2} \operatorname{Pr}^{-1} \frac{\partial w}{\partial t}=-\frac{\partial p}{\partial z}+\epsilon^{2} \triangle_{2} w+\frac{\partial^{2} w}{\partial y^{2}}+R a\left[1+\alpha\left(\cos \left(\Omega_{1} t\right)+\cos \left(\Omega_{2} t\right)\right)\right] T \\
& \epsilon^{2} \operatorname{Pr}^{-1} \frac{\partial T}{\partial t}=\epsilon^{2} w+\epsilon^{2} \triangle_{2} T+\frac{\partial^{2} T}{\partial y^{2}}
\end{aligned}
$$

where $\triangle_{2}=\partial^{2} / \partial x^{2}+\partial^{2} / \partial z^{2}, R a=\beta g \triangle T e^{2} d / \nu \kappa$ is the gravitational Rayleigh number of the cell, $\Omega_{i}=\omega_{i} d^{2} / \kappa(i=1,2)$ are two dimensionless frequencies, and $\alpha=b_{1} \omega_{1}^{2} / g=b_{2} \omega_{2}^{2} / g$ represents the amplitudes ratio of the acceleration of the oscillatory motion to the acceleration of gravity. Moreover, on the vertical walls, the boundary conditions are: $\mathbf{V}=0$ and $\partial T / \partial y=0$ at $y= \pm 1 / 2$. The boundary conditions on the horizontal plates will be discussed below. 


\section{$3 \quad$ Asymptotic study and stability}

In the Hele-Shaw approximation, the order of magnitude of the Prandtl number $\operatorname{Pr}$ in the system of equations (4)-(8) has been discussed in [29]. In what follows, we distinguish two different formulations depending on the order of magnitude of the Prandtl number.

\section{1 $\operatorname{Pr}=O(1)$ or $\operatorname{Pr} \gg 1$}

In this formulation, a first approximation is obtained from the system of equations (4)-(8) by setting $\epsilon^{2}=0$. Remark that in this situation, the term $\partial \mathbf{V} / \partial t$ disappears. Let us denote by $u_{o}, v_{o}, w_{o}, p_{o}$ and $T_{o}$ the solution of such approximation. From equation (6), the pressure is independent of $y$. Also, from equation (8) and using the adiabatic condition, the temperature $T_{o}$ is also independent of $y$. Thus, the system (4)-(8) becomes

$$
\begin{aligned}
& \frac{\partial u_{o}}{\partial x}+\frac{\partial v_{o}}{\partial y}+\frac{\partial w_{o}}{\partial z}=0 \\
& \frac{\partial^{2} u_{o}}{\partial y^{2}}=\frac{\partial p_{o}}{\partial x} \\
& \frac{\partial^{2} w_{o}}{\partial y^{2}}=\frac{\partial p_{o}}{\partial z}-R a\left[1+\alpha\left(\cos \left(\Omega_{1} t\right)+\cos \left(\Omega_{2} t\right)\right)\right] T_{o}
\end{aligned}
$$

Integrating equations (10) and (11), we obtain $u_{o}$ and $w_{o}$. Substituting $u_{o}$ and $w_{o}$ into the continuity equation (9) and integrating, $v_{o}$ is determined. The solution $v_{o}$ which satisfies the boundary condition $v_{o}=0$ at $y= \pm \frac{1}{2}$ is $v_{o}=0$. At the first order $\left(\epsilon^{2}=0\right)$, equations $(10)$ and (11) cannot be coupled to the energy equation (8). The situation corresponds to a pseudosingular perturbation. Therefore, the energy equation (8) is exploited at the order $\epsilon^{2}$ by using the accurate expansion

$$
w=w_{o}+\epsilon^{2} w_{1}, \quad T=T_{o}+\epsilon^{2} T_{1}
$$

Inserting expression (12) into equation (8) and keeping only terms of order $\epsilon^{2}$, one obtains

$$
\frac{\partial T_{o}}{\partial t}=w_{o}+\triangle_{2} T_{o}+\frac{\partial^{2} T_{1}}{\partial y^{2}}
$$

in which $T_{1}$ must also verify the adiabatic condition on the vertical walls: $\frac{\partial T_{1}}{\partial y}=0$ at $y=$ $\pm \frac{1}{2}$. In contrast to the original system (4)-(8), which needs six boundary conditions at the horizontal walls, the system to the first-order (10)-(11) and (13), resulting from the Hele-Shaw approximation, requires only the relevant four boundary conditions

$$
w_{o}=T_{o}=0 \text { at } z= \pm \frac{1}{2}
$$


To perform a stability analysis, we seek the solution of systems (10)-(11), (13) and (14) in term of normal modes as

$$
\begin{aligned}
& u_{o}=\pi g(t)\left(y^{2}-\frac{1}{4}\right) \sin (\pi z) e^{i q x} \\
& w_{o}=i q g(t)\left(y^{2}-\frac{1}{4}\right) \cos (\pi z) e^{i q x} \\
& T_{o}=i q f(t) \cos (\pi z) e^{i q x}
\end{aligned}
$$

where $q$ denotes the wave number, $f(t)$ and $g(t)$ designate, respectively, the amplitudes of the temperature and the velocity field. Using the above assumptions, the system (10)-(11), (13) and (14) is reduced to the differential equation

$$
\dot{f}+\frac{h}{12}\left[R_{o}-R a\left(1+\alpha\left(\cos \left(\Omega_{1} t\right)+\cos \left(\Omega_{2} t\right)\right)\right)\right] f=0
$$

where $h=\frac{\lambda}{(\lambda+1)}, \lambda=\frac{q^{2}}{\pi^{2}}$ and $R_{o}=12 \pi^{2} \frac{(\lambda+1)^{2}}{\lambda} ; R_{o}$ is the Rayleigh number corresponding to the marginal stability of the unmodulated case [30]. The solution of equation (16) is given by

$$
f(t)=f_{o} e^{-\frac{h}{12}\left[\left(R_{o}-R a\right) t-(\alpha R a)\left(\frac{\sin \left(\Omega_{1} t\right)}{\Omega_{1}}+\frac{\sin \left(\Omega_{2} t\right)}{\Omega_{2}}\right)\right]}
$$

where $f_{o}$ is an arbitrary constant depending on the initial conditions. If the fluid layer is heated from below (i.e $R a>o$ ), we can see from equation (17) that the stability criterion is $\left(R a<R_{o}\right)$. This criterion is the same that the one corresponding to the unmodulated case: $R a_{c}=48 \pi^{2}$ and $q_{c}=\pi[30]$. Nevertheless, at the onset of convection, the amplitudes of temperature and velocity field are quasi-periodic. These amplitudes are given by

$$
\begin{aligned}
f(t) & =f_{o} e^{-2 \pi^{2} F r_{1} \Omega_{1}\left(\sin \left(\Omega_{1} t\right)+\frac{\sin \left(\Omega_{2} t\right)}{\omega}\right)} \\
g(t) & =-12 \pi^{2} f_{o}\left[1+F r_{1} \Omega_{1}^{2}\left(\cos \left(\Omega_{1} t\right)+\cos \left(\Omega_{2} t\right)\right] e^{-2 \pi^{2} F r_{1}\left[\sin \left(\Omega_{1} t\right)+\frac{\sin \left(\Omega_{2} t\right)}{\omega}\right]}\right.
\end{aligned}
$$

where $\omega=\Omega_{2} / \Omega_{1}$ and $F r_{1}=\frac{(\kappa / d)^{2}}{g d} \frac{b_{1}}{d}$ represents a sort of Froude number. In this case, the quasi-periodic excitation has neither a stabilizing nor a destabilizing one. On the other hand, if the fluid layer is heated from above (i.e $R a<0$ ), the rest state of the fluid layer cannot be destabilized. We can conclude in this first formulation that if the fluid has a Prandtl number $\operatorname{Pr}=O(1)$ or $\operatorname{Pr}>>1$, the oscillation of the cell cannot generate convective quasi-periodic parametric instabilities. The physical reason is related to the large friction at the vertical walls which leads to the suppression of inertial effects. Quite the same situation takes place in the case of a porous media when the Darcy law is assumed for the resistance force.

\section{$3.2 P r=O\left(\epsilon^{2}\right)$}

In this formulation the term $\partial \mathbf{V} / \partial t$ remains in equations (5) and (7) after making the HeleShaw approximation $\left(\epsilon^{2}=0\right)$. Hence, setting $\operatorname{Pr}=\epsilon^{2} P r^{*}$ where $P r^{*}=O(1)$, system (4)-(8) becomes

$$
\frac{\partial u_{o}}{\partial x}+\frac{\partial v_{o}}{\partial y}+\frac{\partial w_{o}}{\partial z}=0
$$




$$
\begin{aligned}
& \operatorname{Pr}^{*-1} \frac{\partial u_{o}}{\partial t}=-\frac{\partial p_{o}}{\partial x}+\frac{\partial^{2} u_{o}}{\partial y^{2}} \\
& \operatorname{Pr}^{*-1} \frac{\partial w_{o}}{\partial t}=-\frac{\partial p_{o}}{\partial z}+\frac{\partial^{2} w_{o}}{\partial y^{2}}+R a\left[1+\alpha\left(\cos \left(\Omega_{1} t\right)+\cos \left(\Omega_{2} t\right)\right)\right] T_{o} \\
& \frac{\partial T_{o}}{\partial t}=w_{o}+\triangle_{2} T_{o}+\frac{\partial^{2} T_{1}}{\partial y^{2}}
\end{aligned}
$$

where $p_{o}=p_{o}(x, z)$ and $T_{o}=T_{o}(x, z)$. As previously, the energy equation (21) is obtained at the order $\epsilon^{2}$, in which the term $T_{1}$ verifies the adiabatic conditions on the vertical walls: $\partial T_{1} / \partial y=0$ at $y= \pm \frac{1}{2}$. The solution of the system (18)-(21) can be sought in forms (15). Using (18) we find that $v_{o}=0$ and then, after substituting expressions (15) into (19)-(21) and averaging with respect to $y$, we obtain the quasi-periodic differential equation

$$
\frac{d^{2} f}{d t^{2}}+2 p \frac{d f}{d t}+h \operatorname{Pr}^{*}\left[R_{o}-R a\left(1+\alpha\left(\cos \left(\Omega_{1} t\right)+\cos \left(\Omega_{2} t\right)\right)\right)\right] f=0
$$

where $2 p=\pi^{2}+q^{2}+12 P r^{*}$. Now we shall to analyze the stability of the equilibrium state using the amplitude equation of temperature (22). The change of variable $\tau=\Omega_{1} t$ reduces equation (22) to the quasi-periodic Mathieu equation studied by Rand et al. $[27,28]$

$$
\frac{d^{2} f}{d t^{2}}+2 \mu \frac{d f}{d t}+[\delta+\epsilon(1+\alpha(\cos (\tau)+\cos (\omega \tau)))] f=0
$$

where $\mu=p / \Omega_{1}, \delta=h \operatorname{Pr}^{*}\left(R_{0}-R a\right) / \Omega_{1}^{2}, \epsilon=-h \operatorname{Pr}^{*} \operatorname{Ra} F r, \omega=\Omega_{2} / \Omega_{1}$. As noticed before, the Floquet theory cannot be used to determine solutions of equation (23). Following Rand et al. $[27,28]$, we use the harmonic balance method to determine the marginal stability curves by means of expansion

$$
f(t)=\sum_{n=0}^{\infty} \sum_{m=-\infty}^{\infty}\left[A_{n m} \cos \left(\frac{n+m \omega}{2} t\right)+B_{n m} \sin \left(\frac{n+m \omega}{2} t\right)\right]
$$

Approximate results are obtained by a truncation of the infinite sums in equation (24) and then replaced by sums from 0 to $N$ for $n$ and from $-N$ to $N$ for $m$ respectively. In the case, $N=1$ $(n=0,1 ; m=-1,0,1)$, equations (23) and (24) allow us to obtain two homogenous algebraic systems in $A_{n m}$ and $B_{n m}$ which verify: $A_{-n,-m}=A_{n, m}$ and $B_{-n,-m}=-B_{n, m}$. The first system in $A_{n m}$ is in the form

$$
\left\{\begin{array}{l}
\left(\delta-\frac{\omega^{2}}{4}\right) A_{0,1}+\mu \omega B_{0,1}+\frac{\epsilon}{2} A_{0,1}=0 \\
\delta A_{0,0}=0 \\
\left(\delta-\frac{(1-\omega)^{2}}{4}\right) A_{1,-1}+\mu(1-\omega) B_{1,-1}+\epsilon A_{1,1}=0 \\
\left(\delta-\frac{1}{4}+\frac{\epsilon}{2}\right) A_{1,0}+\mu B_{1,0}=0 \\
\left(\delta-\frac{(1+\omega)^{2}}{4}\right) A_{1,1}+\mu(1+\omega) B_{1,1}+\epsilon A_{1,-1}=0
\end{array}\right.
$$


The second system in $B_{n m}$ is given by

$$
\left\{\begin{array}{l}
\left(\delta-\frac{\omega^{2}}{4}-\frac{\epsilon}{2}\right) B_{0,1}-\mu \omega A_{0,1}=0 \\
\left(\delta-\frac{(1-\omega)^{2}}{4}\right) B_{1,-1}-\mu(1-\omega) A_{1,-1}=0 \\
\left(\delta-\frac{1}{4}-\frac{\epsilon}{2}\right) B_{1,0}-\mu A_{1,0}=0 \\
\left(\delta-\frac{(1+\omega)^{2}}{4}\right) B_{1,1}-\mu(1+\omega) A_{1,1}=0
\end{array}\right.
$$

Coupling these two systems, we obtain the following system in $A_{n, m}$

$$
\left(\begin{array}{ccccc}
a_{11} & 0 & 0 & 0 & 0 \\
0 & a_{22} & 0 & 0 & 0 \\
0 & 0 & a_{33} & 0 & a_{35} \\
0 & 0 & 0 & a_{44} & 0 \\
0 & 0 & a_{53} & 0 & a_{55}
\end{array}\right)\left(\begin{array}{c}
A_{0,0} \\
A_{0,1} \\
A_{1,-1} \\
A_{1,0} \\
A_{1,1}
\end{array}\right)=0
$$

where

$$
\begin{aligned}
& a_{11}=\delta, \quad a_{22}=-\frac{\frac{\epsilon^{2}}{2}-\left(\delta-\frac{\omega^{2}}{4}\right)^{2}-\mu^{2} \omega^{2}}{\frac{\omega^{2}}{4}-\delta+\frac{\epsilon}{2}} \\
& a_{33}=\frac{\left(\delta-\frac{(1-\omega)^{2}}{4}\right)^{2}+\mu^{2}(1-\omega)^{2}}{\delta-\frac{(1-\omega)^{2}}{4}}, \quad a_{35}=\epsilon \\
& a_{53}=\epsilon, \quad a_{44}=\frac{\left(\delta-\frac{1}{4}\right)^{2}-\frac{\epsilon^{2}}{4}+\mu^{2}}{\delta-\frac{1}{4}-\frac{\epsilon}{2}} \\
& a_{55}=\frac{\left(\delta-\frac{(1+\omega)^{2}}{4}\right)^{2}+\mu^{2}(1+\omega)^{2}}{\delta-\frac{(1+\omega)^{2}}{4}}
\end{aligned}
$$

The system (25) have non-trivial solution if its determinant vanishes. For each $N$, the dimension of this system is $2 N^{2}+2 N+1$. For the case $N=4$ considered in the current paper, the 
corresponding system dimension is equal to 41 . Nevertheless, the analysis is facilitated by putting the system in upper triangular form. We show in Fig. 2 in the plane $(\delta, \omega)$ when $\epsilon=0.1$ and $\mu=0$, the stability chart as obtained by Rand et al. [27,28].

In this analysis, the vanishing determinant can be given formally in the form $F\left(R a, q, \operatorname{Pr}^{*}, F r_{1}\right.$, $\left.\Omega_{1}, \omega\right)=0$ in which all parameters of the physical problem are taken into account. The marginal stability curves, $R a(q)$, are determined numerically by fixing the dimensionless frequency, $\Omega_{1}$, the frequency ratio, $\omega$, the effective Prandtl number, $P r^{*}$ and the Froude number, $F r_{1}$. Hereafter, we focus attention on the curves corresponding to the critical Rayleigh number, $R a_{c}$, and wave numbers, $q_{c}$, versus the dimensionless frequency $\Omega_{1}$.

Figure 3 illustrates the results of the case where the fluid layer is heated from below and for values of frequencies ratio, $\omega=\sqrt{2}$, effective Prandtl number, $P r^{*}=1$, and Froude number, $F r_{1}=1.610^{-4}$. We see that near $\Omega_{1} \sim 0$, the critical Rayleigh and wave numbers tend, respectively, to the values of the unmodulated case, namely $R a_{c}=48 \pi^{2}$ and $q_{c}=\pi$. Here, it turns out that the effect of modulation is stabilizing for all values of $\Omega_{1}$. Furthermore, the critical Rayleigh number reaches the asymptotic value $R a_{c}=1178$ for high frequencies. The evolution of the critical wave number gives rise to two jump phenomena when crossing $\Omega_{1}=314$ and $\Omega_{1}=360$.

Figure 4 shows the evolution of the critical Rayleigh number as a function of $\Omega_{1}$ for $\operatorname{Pr}^{*}=1$, $F r_{1}=1.610^{-4}$ and for different values of the irrational ratio of frequencies, $\omega$. In contrast to the curves corresponding to $\omega=1 / \sqrt{2}, \sqrt{2}, \sqrt{3}, \sqrt{5}, \sqrt{1} 1, \sqrt{1} 7$ and $\omega=\sqrt{3} 7$ where we are always in the presence of a stabilizing effect, the curve corresponding to $\omega=\frac{1}{\sqrt{3} 7}$ gives rise to either a stabilizing or destabilizing effect. For a fixed frequency $\Omega_{1}$, the critical Rayleigh number $R a_{c}$ increases with increasing $\omega$. Also, at high frequencies, the asymptotic values of the critical Rayleigh number increases with increasing $\omega$. These results suggest that the onset of convection is well controlled by varying the frequencies ratio. Note that for values of $\Omega_{1} \leq 360$ approximatively, $\omega$ has no significant effect on the variation of the critical Rayleigh number.

We illustrate in Fig.5 the dependance of the critical Rayleigh number, $R a_{c}$,on the effective Prandtl number, $\operatorname{Pr}^{*}$, for $\Omega_{1}=100, F r_{1}=1.610^{-4}$ and for different values of the irrational frequencies ratio. It can be seen from Fig. 5 that the largest critical Rayleigh number, corresponding to the maximum of stabilization, increases with decreasing $\omega$ and then the stabilizing effect decreases with the frequency ratio. The Prandtl number corresponding to the largest value of $R a_{c}$ increases weakly from $\operatorname{Pr}^{*}=9.3$ for $\omega=\sqrt{3} 7$ to $P r^{*}=11.2$ for $\omega=\sqrt{5}$ and decreases to the value $\operatorname{Pr}^{*}=7.4$ for $\omega=1 / \sqrt{2}$. However, for high values of Prandtl number, the critical Rayleigh number for all the profiles tends, as expected, to the value of the unmodulated case $R a_{c}=48 \pi^{2}$. Indeed, in this situation the inertial term $\operatorname{Pr}^{-1} \frac{\partial}{\partial t}$ disappears from equations (19)-(20).

In Fig.6, we present the results corresponding to the case of a fluid layer heated from above for $P r^{*}=1, F r_{1}=1.610^{-4}$ and for the values $\omega=1 / \sqrt{3} 7, \omega=\sqrt{2}$ and $\omega=\sqrt{3} 7$. This stable configuration is potentially unstable at high frequencies, $\Omega_{1}$, than with the small ones. Indeed, for each frequency ratio $\omega$, as $\Omega_{1}$ tends to zero, the critical Rayleigh number increases to high values (stable equilibrium configuration) and decreases with increasing $\Omega_{1}$ to reach an asymptotic value. We notice that, as in the case of heating from below, the asymptotic critical Rayleigh number decreases with decreasing $\omega$. The evolution of the critical wave number $q_{c}$ for $\omega=\sqrt{2}$ 
gives rise to two jump phenomena when crossing $\Omega_{1}=296$ and $\Omega_{2}=448$.

\section{Conclusion}

In this work we have studied the effect of a vertical quasi-periodic oscillation on the onset of convection in a Hele-Shaw cell. We have considered the case of a heating from below and the case of a heating from above. Two formulations depending on the order of magnitude of the Prandtl number have been discussed. In the first formulation, $\operatorname{Pr}=O(1)$ or $\operatorname{Pr} \gg 1$, we have obtained, as expected, the same criterion of stability that the one of the unmodulated case. In the second formulation, $\operatorname{Pr}=O\left(\epsilon^{2}\right)$, the governing system of equations is reduced to a damped quasi-periodic Mathieu equation. In this situation, using the harmonic balance method and Hill's determinants, we have determined the onset of convection characterized by a quasi-periodic regime. It was shown that a modulation with two incommensurate frequencies produces a stabilizing or a destabilizing effect strongly depending on the ratio of the frequencies. In summary, the study considered in the second formulation showed that the frequency ratio plays an important role in controlling the onset of convection.

From a physical point of view, our motivation was based on the fact that the Hele-Shaw cells are widely used to simulate two-dimensions flows in porous media [31-35]. Therefore, this linear study can serve as model to investigate the influence of the quasi-periodic gravitational modulation on the threshold of convective instability in porous medium.

\section{References}

[1] P.M. Gresho, R.L. Sani, The effects of gravity modulation on the stability of a heated fluid layer, J. Fluid Mech. 40 (4) (1970) 783-806.

[2] G.Z. Gershuni, E. M. Zhukhovitskii, The Convective Stability of Incompressible Fluids, (Keter Publications, Jerusalem, 1976), 203-230.

[3] M.Lücke, F. Shank, Response to parametric modulation near an instability, Phys. Rev. Lett. 54 (14) (1985) 1465-1468.

[4] M. Wadih, B. Roux, The effects of gravity modulation on the stability of a heated fluid layer, J. Fluid Mech. 40 (4) (1970) 783-806.

[5] S. Biringen, L.J. Peltier, Numerical simulation of 3-D Bénard convection with gravitational modulation, Phys. Fluids A2 (1990) 754.

[6] R. Clever, G. Schubert, F.H. Busse, Two-dimensional oscillatory convection in a gravitationally modulated fluid layer, J. Fluid Mech. 253 (1993) 663-680.

[7] R. Clever, G. Schubert, F.H. Busse, Three-dimensional oscillatory convection in a gravitationally modulated fluid layer, Phys. Fluids A5 (10) (1993) 2430.

[8] U.E. Volmar, H.W. Muller, Quasiperiodic patterns in Rayleigh-Bénard convection under gravity modulation, Phys. Rev. E 56 (1997) 5423-5430. 
[9] J.L. Rogers, M.F. Schatz, J.L. Bougie, J.B. Swift, Rayleigh Bénard convection in a vertically oscillated fluid layer, Phys. Rev. Lett. 84 (1) (2000) 87-90.

[10] S. Aniss, M. Souhar, M. Belhaq, Asymptotic study of the convective parametric instability in Hele-Shaw cell, Phys. Fluids 12 (2) (2000) 262-268.

[11] S. Aniss, M. Souhar, M. Belhaq, Effet d'une modulation magnétique sur le seuil d'instabilité convectif au sein d'une couche de liquide magnétique chauffée par le haut, C. R. Mecanique 328 (2000) 399-406.

[12] S. Aniss, M. Belhaq, M. Souhar, Effects of a magnetic modulation on the stability of a magnetic liquid layer heated from above, ASME J. Heat Transfer 123 (2001) 428-432.

[13] B.S. Bhadauria, P.K. Bhatia, L. Debnath, Convection in Hele-Shaw cell with parametric excitation, Int. Journal of Non-Linear Mechanics (40) (2005) 475-484.

[14] G. Venezian, Effect of modulation on the onset of thermal convection, J. Fluid Mech. 35 (2) (1969) 243-254.

[15] S. Rosenblat, D.M. Herbert, Low-frequency modulation of thermal instability, J. Fluid Mech. 43 (2) (1970) 385-398.

[16] S. Rosenblat, G.A. Tanaka, Modulation of thermal convection instability, Phys. Fluids 14 (7) (1971) 1319-1322.

[17] C. S. Yih and C. H. Li, Instability of unsteady flows or configurations. Part 2. Convective instability, J. Fluid Mech. 54 (1972) 143-152.

[18] R.G. Finucane, R.E. Kelly, Onset of instability in a fluid layer heated sinusoidally from below, Int. J. Heat Mass Transfer, 19 (1976) 71-85.

[19] M. N. Roppo, S.H. Davis, S. Rosenblat, Bénard convection with time-periodic heating, Phys. Fluids 27 (4) (1984) 796-803.

[20] G. Ahlers, P.C. Hohenberg, M. Lücke, Externally modulated Rayleigh-Bénard convection: experiment and theory, Phys. Rev. Lett. 53 (1) (1984) 48-51.

[21] P.K. Bhatia, B.S. Bhadauria, Effect of modulation on thermal convection instability, Z. Naturforsch. 55a (2000) 957-966.

[22] P.K. Bhatia, B.S. Bhadauria, Effect of low-frequency modulation on thermal convection instability, Z. Naturforsch, 56a (2001) 509-522.

[23] B.S. Bhadauria, P.K. Bhatia, Time-periodic heating of Rayleigh-Bénard convection, Phys. Scripta 66 (1) (2002) 59-65.

[24] S. Aniss, M. Belhaq, M. Souhar, M.G. Velarde, Asymptotic Study of Rayleigh-Bénard Convection under Time Periodic Heating in Hele-Shaw Cell, Phys. Scripta 71 (4) (2005) 395-401. 
[25] B. Oukada, M.T. Ouazzani, S. Aniss, Effets d'une modulation en phase de température à la frontière sur l'instabilité convective d'une couche liquide viscoélastique, C.R. Mecanique 334 (2006) 205-211.

[26] T. Boulal, S. Aniss, M. Belhaq, R. Rand, Effect of quasiperiodic gravitational modulation on the stability of a heated fluid layer, Phys. Rev. E 76 (52) (2007) 56320.

[27] R. Rand, R.R. Zounes, R. Hastings, Dynamics of a quasiperiodically forced Mathieu oscillator, Chap. 9 in Nonlinear Dynamics: The Richard Rand 50th Anniversary Volume, edited by A. Guran (World Scientific, 1997).

[28] R.S. Zounes, R. Rand, Transition curves of the quasi-periodic Mathieu equation, SIAM J. Appl. Math 58 No 4 (1998) 1094-1115.

[29] S. Aniss, J.P. Brancher, M. Souhar, Asymptotic study and weakly non linear analysis at the onset of Rayleigh-Bénard convection in Hele Shaw cell, Phys. Fluids 7 5, 926 (1995).

[30] H. Frick, R.M. Clever, Einfluss der seitenwande auf das einsetzen der konvection in einer horizontalen flussigkeitsschicht, Z. Angew. Math. Mech. 31, (1980) 502.

[31] J.W. Elder, Steady free convection in a porous medium heated from below, J. Fluid Mech. $72(1967) 30$.

[32] B.K. Hartline, C.R.B Lister, An experiment to verify the permeability of Hele-Shaw cell, Geophys. Res. Lett. 5 (1978) 225.

[33] B.K. Hartline, C.R.B. Lister, Thermal convection in Hele-Shaw cell, J. Fluid Mech. 79 (1977) 379.

[34] O. Kvernvold, On the stability of non linear convection in HeleShaw cell, Int. J. Heat Mass Trans. 22 (1979) 395.

[35] O. Kvernvold, P.A. Tyvand, Nonlinear thermal convection in anisotropic porous media, J. Fluid Mech. 99 (1980) 673. 


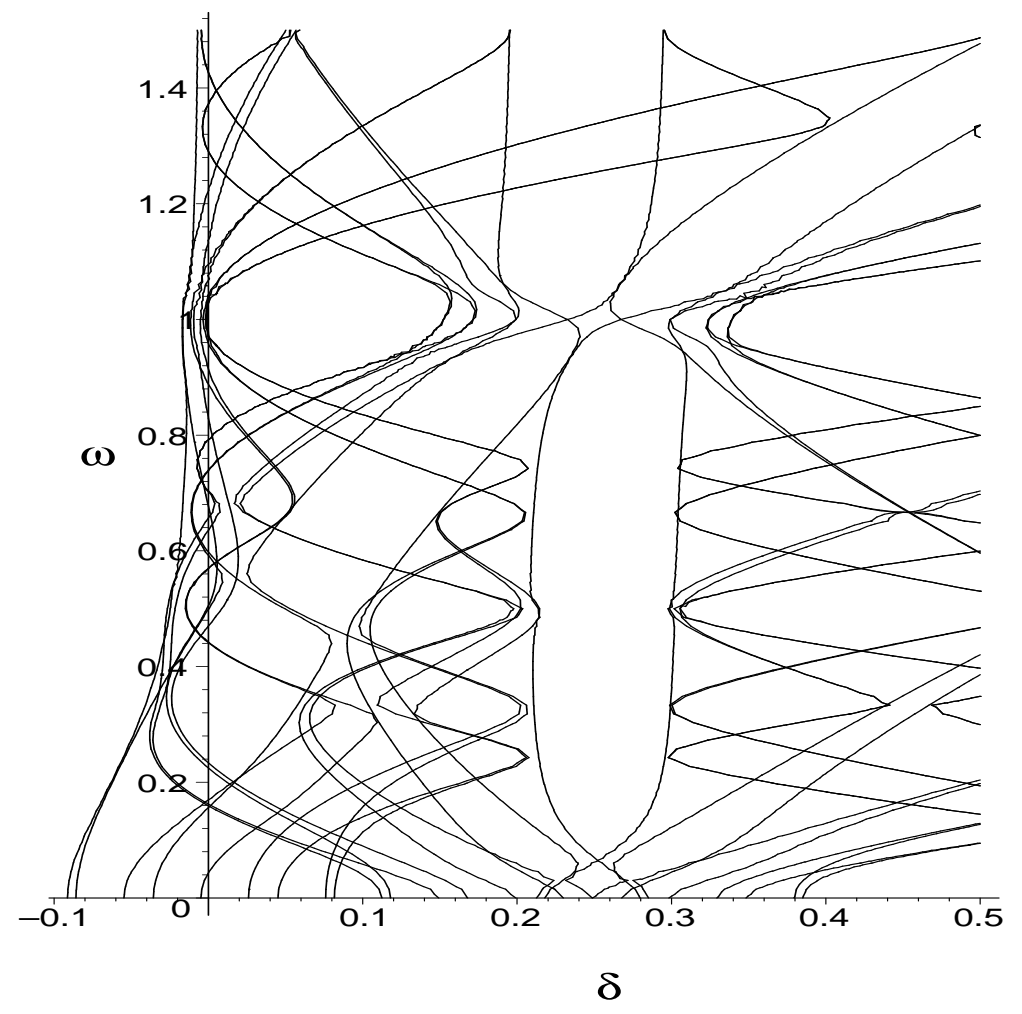

Figure 2: Stability chart of the quasi-periodic Mathieu equation in the plane $(\delta, \omega)$ for $N=4$, $\epsilon=0.1$ and $\mu=0$. 


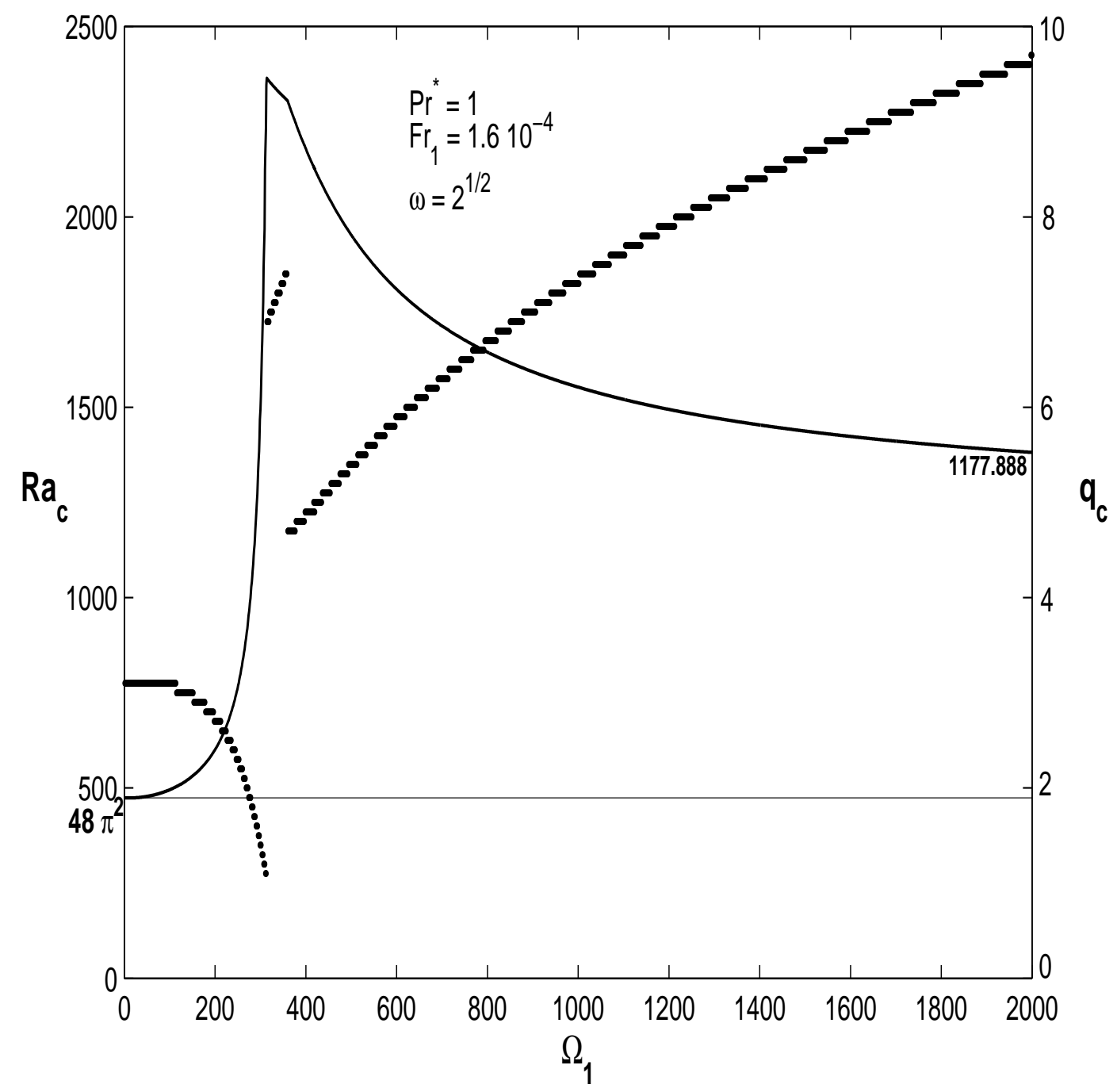

Figure 3: Heating from below - Evolution of the critical Rayleigh number, $R a_{c}$, and wave number, $q_{c}$, as a function of the dimensionless frequency $\Omega_{1}$ for $\omega=\sqrt{2}$. 


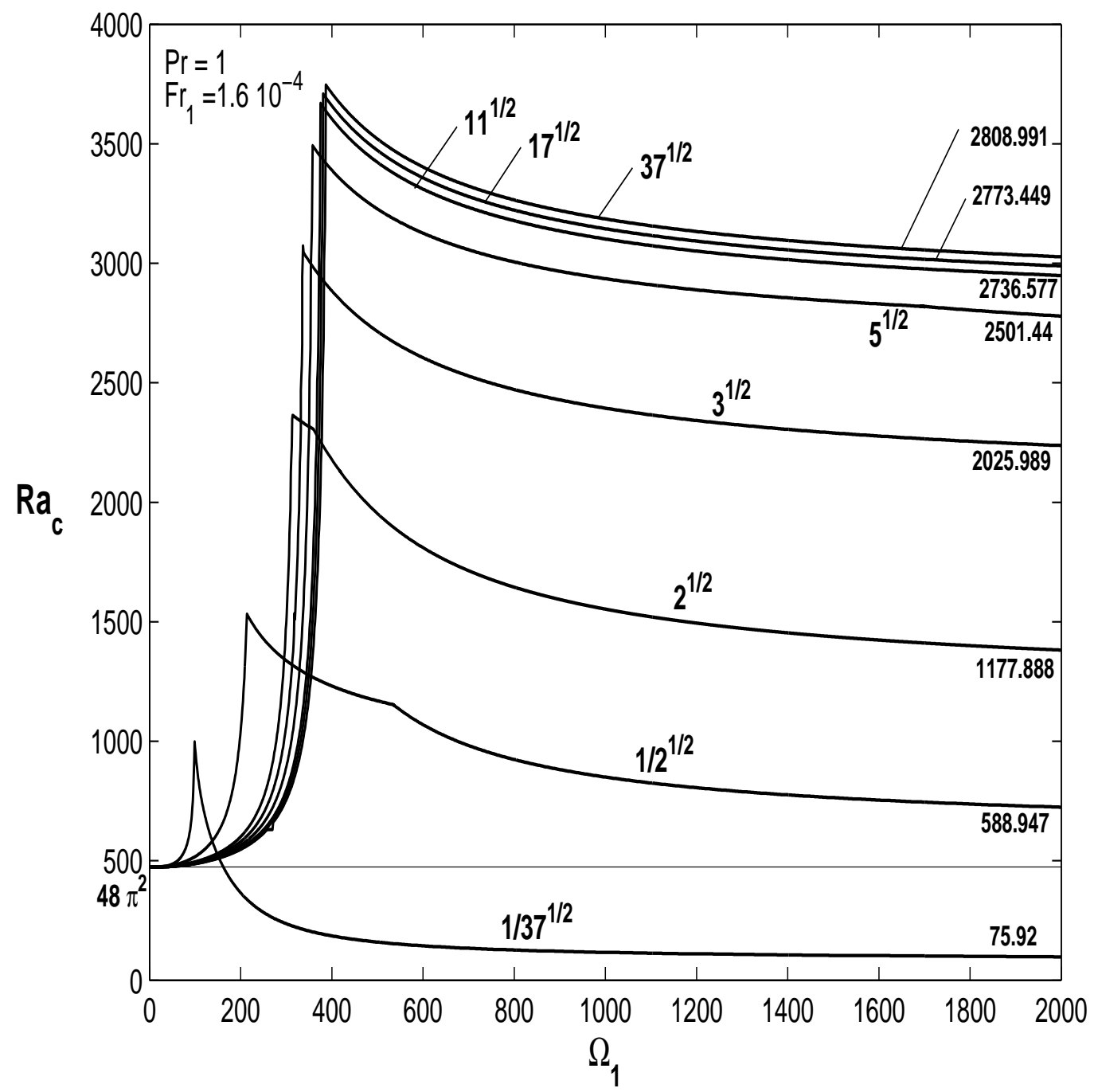

Figure 4: Heating from below - Evolution of the critical Rayleigh number, $R a_{c}$, as a function of the nondimensional frequency, $\Omega_{1}$, for different values of the irrational frequencies ratio, $\omega$. 


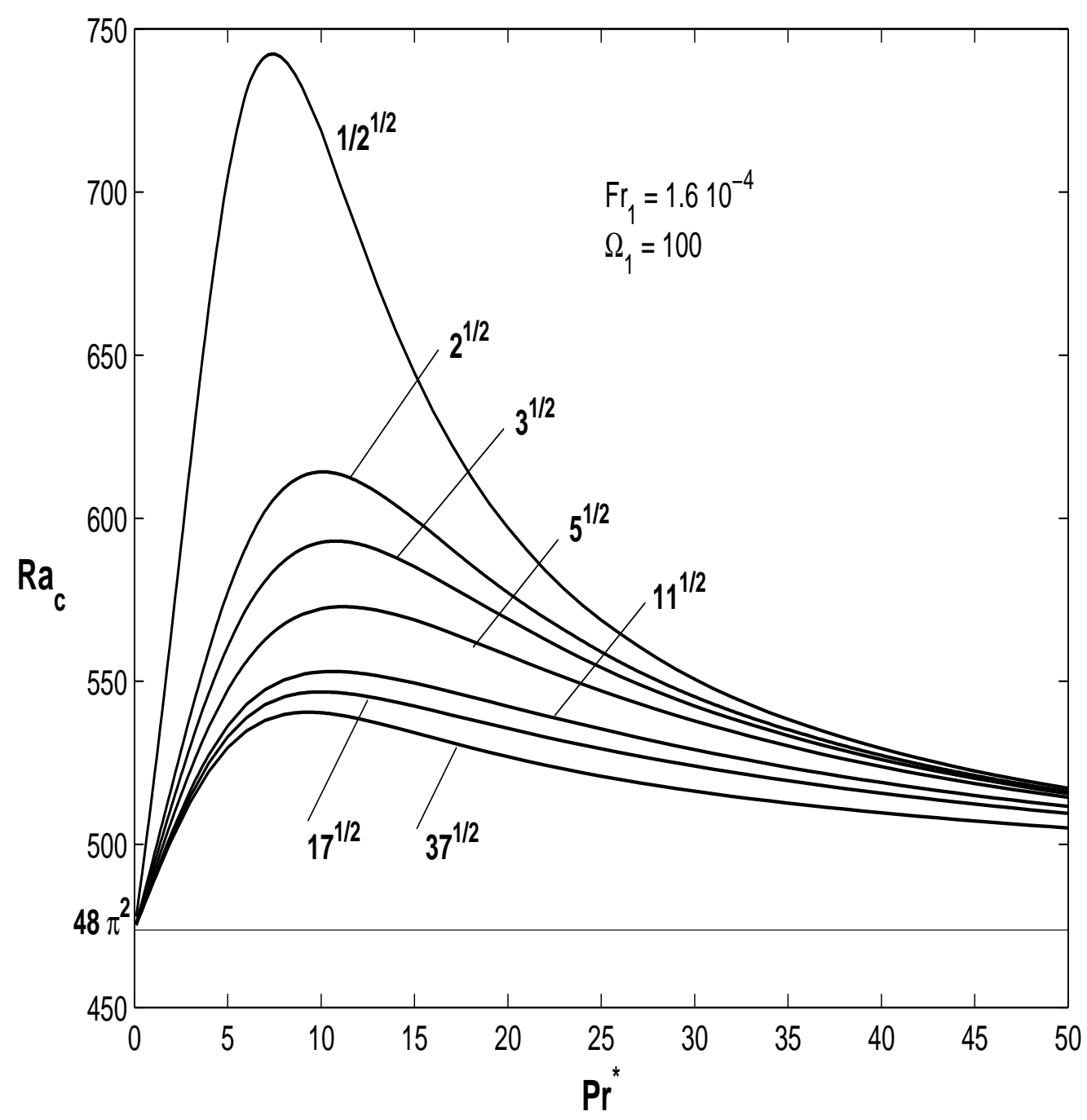

Figure 5: Heating from below - Evolution of the critical Rayleigh number, $R a_{c}$, as a function of the Prandt number, $\operatorname{Pr}$, for $\Omega_{1}=100$ and for different values of the irrational frequencies ratio, $\omega$. 


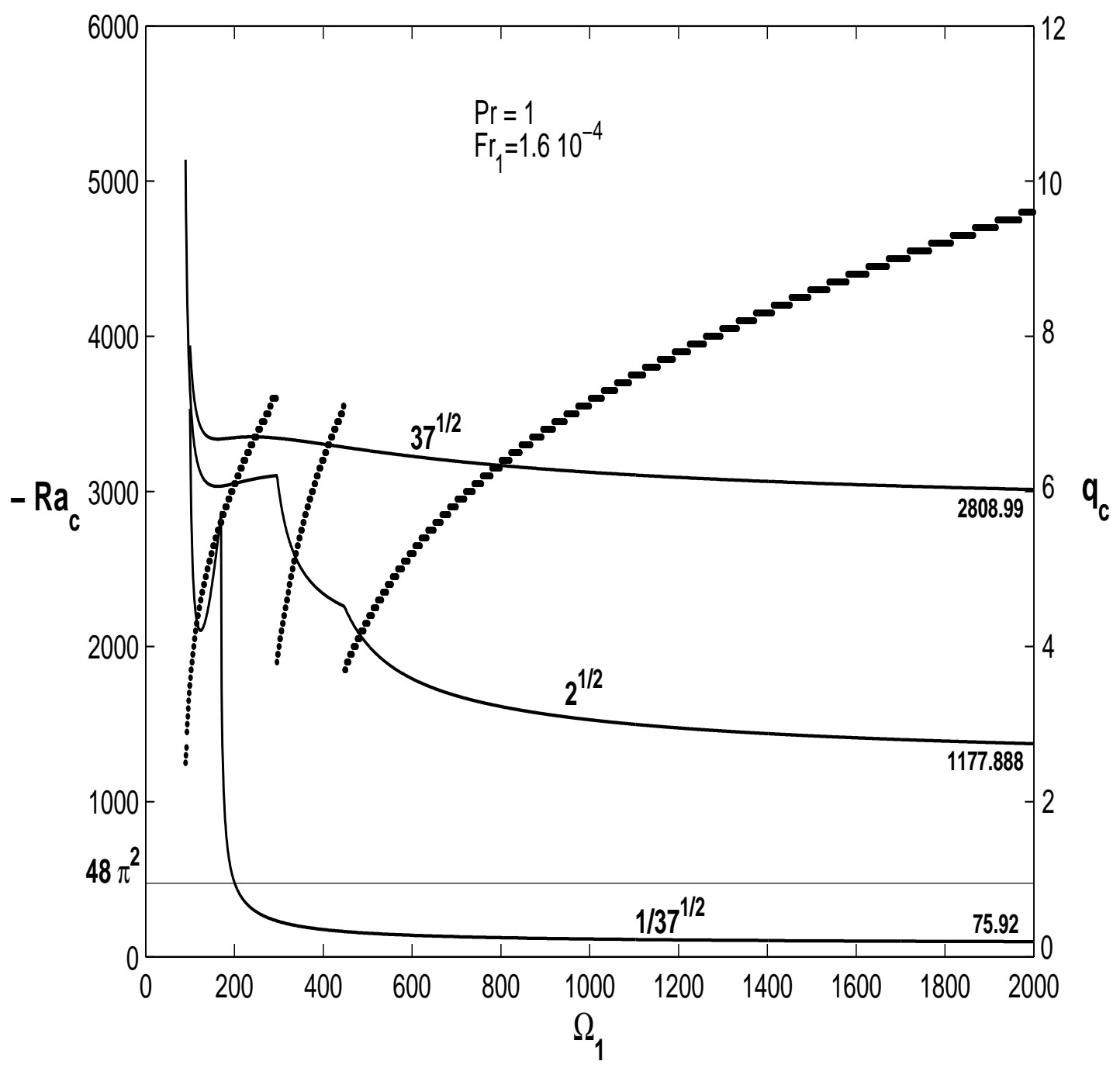

Figure 6: Heating from above - Evolution of the critical Rayleigh number, $R a_{c}$, and wave number, $q_{c}$, as a function of the dimensionless frequency $\Omega_{1}$. 